\title{
Analysis of Intermediate Hosts and Susceptible Animals of SARS-CoV-2 by Computational Methods
}

\author{
Yueying Yang ${ }^{1, \#,}$ Mengzhu Zheng ${ }^{2, \#}$, Yang Liu ${ }^{1}$, Yali Wang ${ }^{1}$, Yang $\mathrm{Xu}^{1}{ }^{1}$, Yirong Zhou $^{2}$, \\ Dejuan Sun ${ }^{1}$, Lixia Chen ${ }^{1, *}$ and Hua $\mathrm{Li}^{1,2, *}$
}

\begin{abstract}
COVID-19, a disease caused by SARS-CoV-2 that produces major symptoms of pneumonia, has been a disaster worldwide. The traceability of SARSCoV-2 and the discovery of susceptible animal species is crucial to halt viral transmission and explore the mechanism of cross-species transmission. We selected 82 representative ACE2 sequences from the 1000 sequences with the closest homology to the hACE2 protein. All selected ACE2 proteins were subjected to homology modeling. Potential natural and intermediate hosts, as well as animal species susceptible to SARS-CoV-2, were analyzed systematically by calculation of the binding free energy of ACE2 protein to the RBD of SARSCoV-2. Primates, some wild Felidae, civets, goats, spotted hyenas and golden hamsters are susceptible to SARS-CoV-2 and may be potential intermediate hosts, whereas pangolins, birds and reptiles are unlikely to be intermediate hosts. Mice, rats and guinea pig are not susceptible to SARS-CoV-2. Given their possible susceptibility, non-human primates, goats and golden hamsters could potentially be used as experimental models to examine SARS-CoV-2 infection without transgenesis. Herein, possible candidates for the natural and intermediate hosts of SARS-CoV-2 are suggested, to provide guidance for subsequent studies.
\end{abstract}

Key words: SARS-CoV-2, Intermediate hosts, Susceptible animals, Spike, ACE2

\section{BACKGROUND}

The world is undergoing a battle against the novel coronavirus (SARS-CoV-2). By the end of July 2021, more than 200 million cases of COVID-19 and 4.25 million deaths had occurred worldwide. The pandemic has affected 212 countries and regions globally. Inevitably, all humanity must work together to overcome this obstacle. Since entering the 21st century, humans have experienced three outbreaks of pneumonia due to coronavirus, thus providing a strong reminder that we must pay sufficient attention to coronavirus prevention and treatment. Tracing the origin of SARS-CoV-2 and its route of transmission is important for the development of treatment and prevention strategies for future recurrent epidemics.

According to the transmission route of the virus, hosts are generally divided into natural hosts, intermediate hosts and final hosts. The intermediate hosts of a virus may include multiple species, which act as a vehicle that "transports" the virus from the natural host to the final host. To control further spread of a virus, beyond
Edited by:

Cao Chen, National Institute for

Viral Disease Control and Prevention,

Chinese Center for Disease Control and Prevention

Reviewed by:

Two reviewers request to be anonymous

*Corresponding authors:

E-mail: li_hua@hust.edu.cn,

Tel: +86-27-83692762 (HL),

syzyclx@163.com (LC)

1Wuya College of Innovation, Key Laboratory of Structure-Based Drug Design \& Discovery, Ministry of Education, Shenyang Pharmaceutical University, Shenyang 110016, China ${ }^{2}$ Hubei Key Laboratory of Natural Medicinal Chemistry and Resource Evaluation, School of Pharmacy, Tongji-Rongcheng Center for Biomedicine, Tongji Medical College Huazhong University of Science and Technology, Wuhan 430030, China

\#These authors contributed equally to this work.

Received: August 192021 Revised: August 252021

Accepted: August 262021

Published Online: September 42021 
isolating and treating already infected people, the discovery and isolation of intermediate hosts can actually block the infection from the source. Palm civets may be an intermediate host of SARS-CoV [1], and dromedary camels may be an intermediate host of MARS-CoV [2], both of which have been demonstrated to have originated from bats [3-5]. Shi ZL, et al. have reported a 96.2\% sequence similarity of SARS-CoV-2 and the bat coronavirus RaTG13 (bat-CoV-RaTG13) carried by Rhinolophus affinis in Yunnan Province, China. Furthermore, the sequence similarity of the $S$ gene (encoding the spike protein) of SARS-CoV-2 and bat-CoV-RaTG13 is $93.1 \%$, a value much higher than those with other SARS-CoVs [6].

Currently, research on intermediate hosts of SARS$\mathrm{CoV}-2$ is underway, and the investigated animals include pangolins, minks and turtles. In four studies, the genome sequence similarity between pangolin-CoVs and SARSCoV-2 has been reported to be $85.5 \%$ to $92.4 \%$ [7], 91.02\% [8], 90.3\% [9], and 90.23\% [10]. Two species of SARS$\mathrm{CoV}-2$ related pangolin-CoVs are known: pangolin-CoV GD and pangolin-CoV GX. Researchers have found that although SARS-CoV-2 is closest to bat-CoV-RaTG13 in other regions, SARS-CoV-2 has a high sequence similarity with the receptor binding domain of pangolin-associated coronaviruses. One study has shown that pangolin-CoV GD exhibits strong similarity to SARS-CoV-2 in the receptor-binding domain, and $97.4 \%$ amino acid sequence similarity, a value higher than that of bat-CoV-RaTG13 (89.2\%) [7]. Three studies have supported this result, showing that the RBD is highly conserved between pangolin-CoV GD and SARS-CoV-2, with only a one amino acid residue difference [8-10]. Furthermore, pangolin-CoVs and SARS-CoV-2 have the same amino acids at five key residue positions in the RBD, whereas bat-CoV-RaTG13 has only one amino acid residue consistent with the SARS$\mathrm{CoV}-2$ sequence $[7,8]$. Researchers have also suggested that the amino acid similarity between the pangolin-associated coronavirus RBD and SARS-CoV-2 may be due to selectively mediated convergence during evolution rather than recombination.

However, the SARS-CoV-2 spike protein has a special "PRRA" motif insertion at the S1/S2 cleavage site $[7,8,10,11]$, and this motif is not found in bat-CoV-RaTG13 or pangolin-CoVs. Chen J, et al. have suggested that this motif might have been inserted in other intermediate hosts during viral transmission [10]. Therefore, determining whether pangolins are intermediate hosts of SARS-CoV-2 will require many additional experimental samples and data analysis. Zhu $\mathrm{H}$, et al. have found that mink coronavirus shows an infection pattern closer to that of SARS-CoV-2 according to deep learning algorithms, thus suggesting that minks might be an intermediate host of SARS-CoV-2 [12]. Moreover, another study has suggested that turtles might be intermediate hosts of SARS-CoV-2 [13].

At present, the intermediate host of SARS-CoV-2 has not been determined, and most researchers believe that more than one intermediate host exists. Other researchers believe that intermediate hosts might not be necessary, and the virus can directly infect humans. Most studies have performed identity analysis of genomic sequences only between the potential intermediate host and SARS-CoV-2, and have conducted similarity analysis of some protein domains. No research team is currently conducting experimental verification.

Here, we selected the angiotensin-converting enzyme 2 (ACE2) sequences from other species with the closest homology to the hACE2 protein, including primates, Chiroptera, Felidae, Canidae, Circetidae, Camelidae, and the previously reported Manis javanica and Mustela putorius furo. These species were divided into different families on the basis of sequence alignment, phylogenetic tree analysis and homology modeling of all ACE2 proteins. Protein-protein docking of the SARS-CoV-2 spike with ACE2 from different species and calculations of the binding free energy were performed to identify potential intermediate hosts or animal species susceptible to SARS-CoV-2. In addition, two coronavirus spike proteins with the highest similarity to the SARS-CoV-2 spike were modeled, then docked with hACE2 and various ACE2 proteins to calculate the free energy, to determine the possibility of these coronaviruses directly infecting humans and other animals. We thus used a new approach for mining intermediate hosts and systematically analyzing the potential natural and intermediate hosts of SARS-CoV-2 by calculating the binding free energy between RBD and ACE2. We also provide suggestions for the selection of experimental animals for COVID19 research.

\section{METHODS}

\section{Homology ACE2 protein BLAST searching and sequence alignment}

Amino acid sequence editing was conducted in Bioedit and DNAMAN, and sequence alignment was conducted with Clustalw. The evolutionary history was inferred with the neighbor-Joining method in the MEGA 7 software package. The percentage of replicate trees in which the associated taxa clustered in the bootstrap test was determined with 1000 replicates. Subsequently, 3D structures were analyzed with the PyMOL tool.

The full length ACE2 sequence (NP_001358344.1) was downloaded from the NCBI protein database. The amino acid sequences were aligned with sequences in the entire database with BLASTp to search for homology to the ACE2 protein (algorithm parameters: maximum target sequences: 1000, expected threshold: 10). Accession numbers of the 82 chosen ACE2 sequences are listed in Table 2.

\section{Homology modeling and molecular docking}

On the basis of the recently reported structure of the SARSCoV-2 spike RBD-ACE2 complex (PDB code: 6LZG) [14], corresponding homology models of each spike RBD and ACE2 were built. Alignment of two protein sequences and subsequent homology modeling were performed with the bioinformatics module of ICM 3.7.3 modeling software on 
an Intel i7 4960 processor (MolSoft LLC, San Diego, CA) [15]. Protein-protein docking was performed according to the ICM-Pro manual, and the free binding energy was calculated. The receptors were each ACE2 homologues, and the ligands were each CoV-RBDs. The epitopes of both ACE2 and RBD were selected near the interface of the complex, with the SARS-CoV-2 spike RBD-ACE2 complex crystal structure (PDB code: 6LZG) used as the reference.

\section{RESULTS}

\section{Bioinformatics analysis of ACE2 proteins}

SARS-CoV-2 uses ACE2 as the cellular receptor to invade host cells in a species-dependent manner, as directly reflected in the binding affinity and specificity of the spike RBD and host ACE2. Consequently, ACE2 usage is a crucial determinant of infectivity and host range. Therefore, we collected 1000 hACE2 homologous protein sequences with the BLASTp method. ACE2 sequences from 82 species were chosen, and phylogenetic tree analysis was performed (Fig 1). The 82 species mainly belonged to Mammalia, and several were from other classes, such as Aves, Reptilia and Sauropsida. The mammalian group included primates, rodents, odd-toed hoofed mammals, artiodactyls, carnivores, lagomorphs and bats. Bats have been proposed to be the natural host of SARS-CoV-2 [16]. To identify possible source hosts, we collected all available ACE2 sequences from Chiroptera (total number of 17).

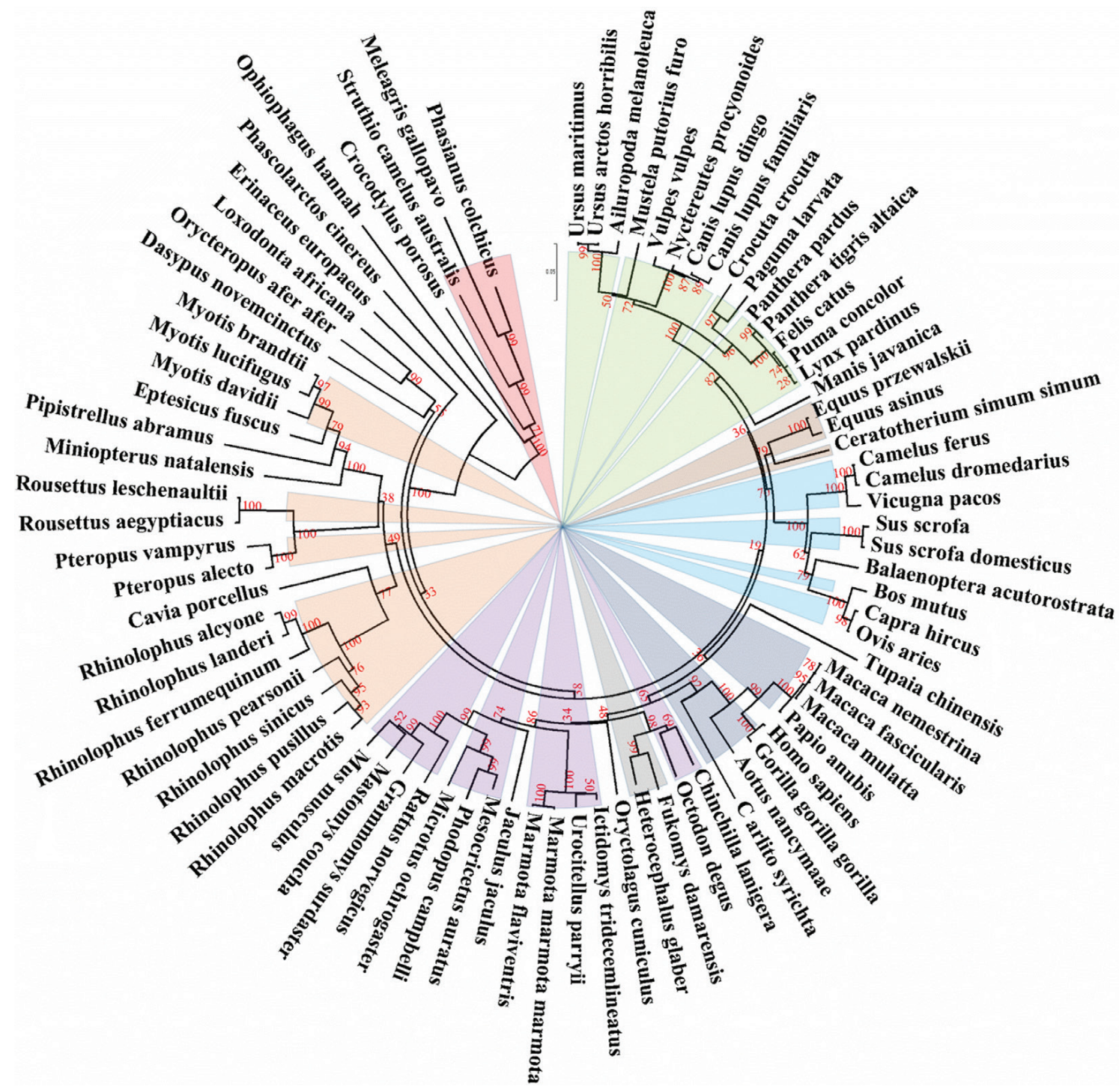

FIGURE 1 | Phylogeny of the complete ACE2 protein sequences from 82 species.

The evolutionary history was inferred with the neighbor-joining method. The optimal tree with a branch length sum of 3.96647534 is shown. The tree is drawn to scale, with branch lengths in the same units as those of the evolutionary distances used to infer the phylogenetic tree. The evolutionary distances were computed with the Poisson correction method and are in units of the number of amino acid substitutions per site. The analysis involved 82 ACE2 amino acid sequences from different species. All positions containing gaps and missing data were eliminated. The final dataset included 625 positions. Evolutionary analyses were conducted in MEGA7. Species included in the same circular sector are from the same family. Species from the same order are marked with a circular sector in the same color. Those that were not marked species came from separate orders. 
The structure of the hACE2 and SARS-CoV-2 spikeRBD complex has been solved [14,17], as shown in Fig 2A and $\mathrm{B}$. The seven amino acids at the hACE2 binding interface and spike-RBD form eight hydrogen bonding interactions: Gln24, Asp30, His34, Tyr41 and Gln42 in hACE2 form hydrogen bonding interactions with Gln474,
A

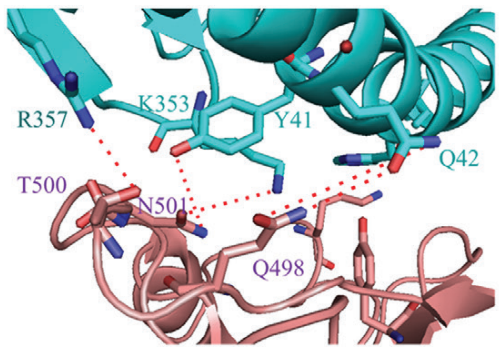

D

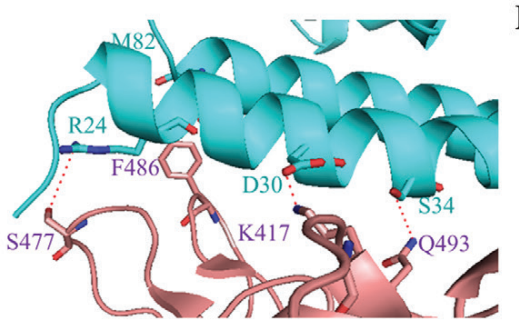

$\mathbf{G}$

\begin{tabular}{|c|c|}
\hline & 40 \\
\hline Homo sapiens & E E QAKTFLDKF NHEAEDLFYQS \\
\hline Gorilla gorilla gorilla & E EQAKT F L D K F NHEA \\
\hline Macaca $n$ & EQAKT FLDKFNHEAEDLFYQS \\
\hline Papio & E EQAKT FLDKF NHEAEDLFY S \\
\hline Macaca fas & E EQAKTFLDKFNHEAEDLFY \\
\hline Panthera tigris altaica & EEQAKTFLDKFNHEAEDLFY \\
\hline Macaca mulatta & E ELAKT F L E KF NHE A E EL S Y \\
\hline Puma & E ELAKT F L E KF NHEA E EL S Y \\
\hline Panthe & E ELAKTFLEKF NHEAE ELS Y \\
\hline Lynx & E ELAKT FLEKF NHE AE ELSYQS \\
\hline Mesocricetl & E EQAKT F L D KF NQEAEDLS Y Q S \\
\hline Crocuta cr & E ELAKT F L E K N N E A Q E S YLS \\
\hline Capra & E EQAKT F L E K F I \\
\hline Ovis a & A K T F L E K F \\
\hline & KT F L E K F I \\
\hline Bos & E EQAKT F L E KF NHEAEDLSYQS \\
\hline Rhinolophus sinicu & DRAKT F L D E F N S E E NL S Y Q S \\
\hline & E ELAKT F L E T F NY E A QELS YQS \\
\hline & $\Lambda \quad \Lambda$ \\
\hline
\end{tabular}

B

$\mathbf{E}$
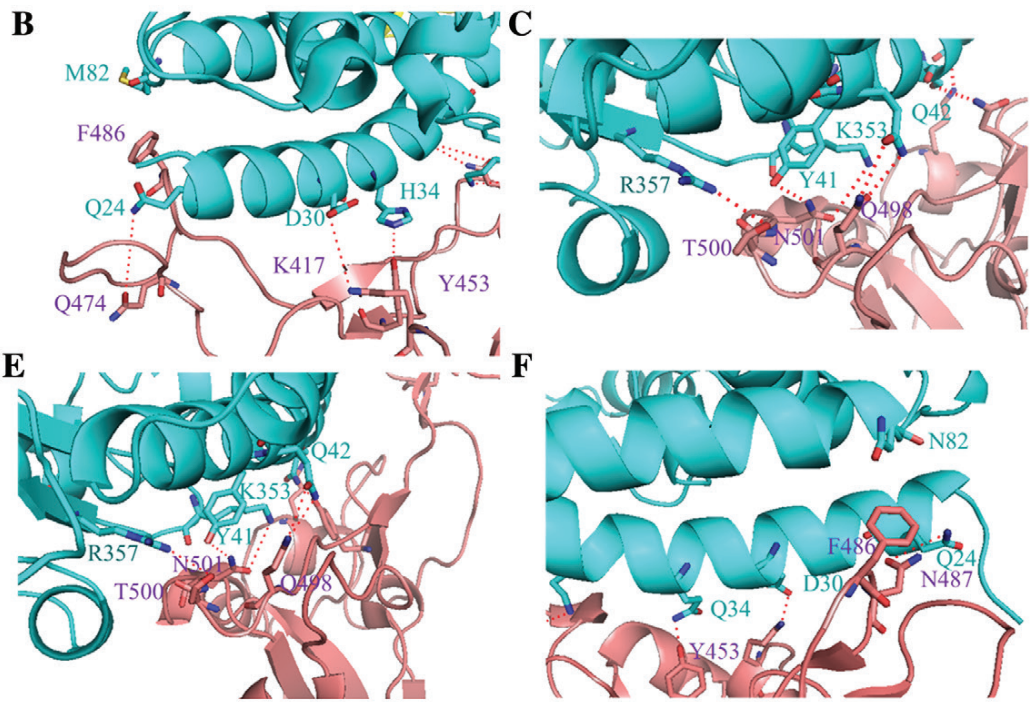

80

L A QMY P

L A QMY P

L A QMY P

L A QMY P

L AQMY P

L A QMY P

L A E T Y P

L A K T Y P

L A E T Y P

L A K T Y P

L AKNY S

L A K T Y P

MART Y S

MAR T Y S

I AKT Y P

MAKT Y S

L AKNY P

L AQT Y P

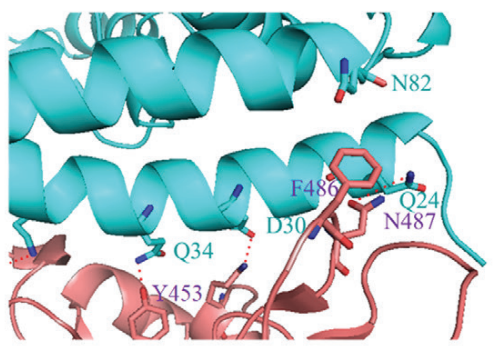

350

360

D L GKGD F R I L MC

D L GK G D F R I L MC

D L GKGD F R I I MC $\Delta$ hydrogen bond

D L GK GD F R I I MC

D L GKGDF R I I MC $\triangle$ Vander Waals

DLGKGDFR I I MC force

DL GKGD F R I KMC

DL GKGD F R I KMC

D L GKG D F R I KMC

DL GKGD F R I KMC

D L GKGD F R I KMC

D L GKGD F R I KMC

DL GKGD F R I KMC

D L GK GD F R I KMC

DL GKGD F R I KMC

D L GK GD F R I KMC

D L GKGD F R I KMC

DL GKGDF R I KMC

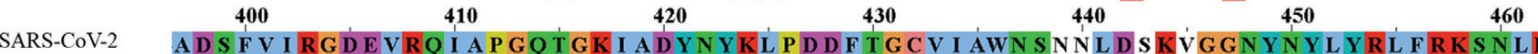

$\begin{array}{ccccc}400 & 410 & 420 & 430 & 40\end{array}$

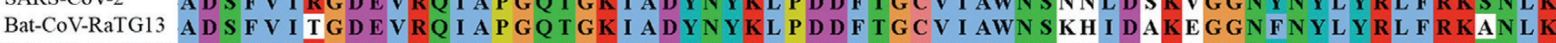

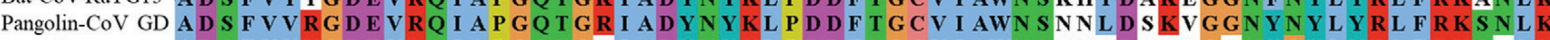
Pangolin-CoV GX A DS F V VKGDEVRQ I A P GQT GV I A DYNYKL P D D F T GCV I AWN S V KQDALTGDNYGYLYRLFRKSKLK

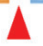

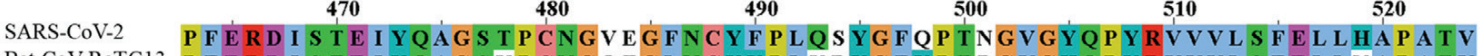

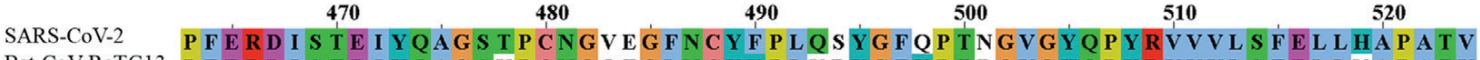

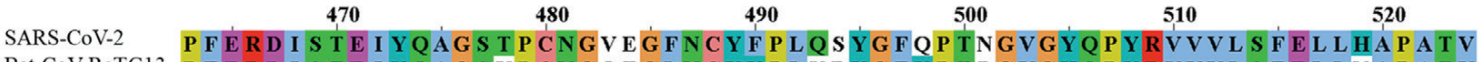

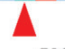

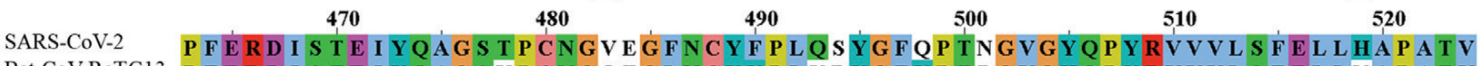

Bat-CoV-RaTG13 P F E R D I S T E I Y QA G S K P C NGQTGL N C Y Y P L Y R Y G F Y P T D G V G H Q P Y R V V VL S F E L L N A P A T V

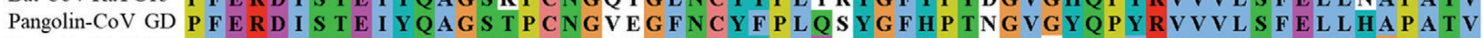
Pangolin-CoV GX P F E R D I S T E I Y QA GS T P C N GQVGL N C Y Y P L E RY G F H P T T G VNY Q P F R V V V L S F E L L H G P A T V

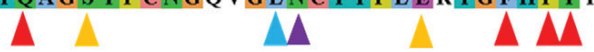

FIGURE 2 | Analysis of key amino acids at the interface of ACE2 and spike-RBD.

$\mathbf{A}$ and $\mathbf{B}$, hACE2 and SARS-CoV-2 spike-RBD interaction interface analysis. These pictures were plotted with PyMOL with the ACE2-spike-RBD complex as a model (PDB code: $6 \mathrm{~m} 17$ ). hACE2 is displayed as a cartoon in blue. SARS-CoV-2 is displayed as a cartoon in brown, and the binding amino acids are displayed as sticks. Red sticks represent oxygen atoms, blue sticks represent nitrogen atoms, and red dotted lines represent hydrogen bonds. C and D. Analysis of the interaction interface between Rhinolophus sinicus ACE2 and the SARS-CoV-2 spike-RBD. $\mathbf{E}$ and $\mathbf{F}$. Analysis of the interaction interface between Mesocricetus auratus ACE2 and the SARS-CoV-2 spike-RBD. G. ACE2 from 18 species that docked with the SARS-CoV-2 spike-RBD with a binding free energy below $-49 \mathrm{~kJ} \mathrm{~mol}^{-1}$. Sequence alignment of the ACE2 spike binding motif, in which amino acids binding the spike-RBD with hydrogen bonds are marked with red triangles below, and amino acids binding the RBD with hydrophobic interactions are marked with blue triangles below. $\mathbf{H}$. Sequence alignment of four coronavirus receptor binding motifs. Amino acids bound to hACE2 with hydrogen bonds are marked with red triangles below. Amino acids predicted to bind Rhinolophus sinicus ACE2 with hydrogen bonds are marked with yellow triangles below. Amino acids with predicted binding to Mesocricetus auratus ACE2 with hydrogen bonds are marked with purple triangles below. 


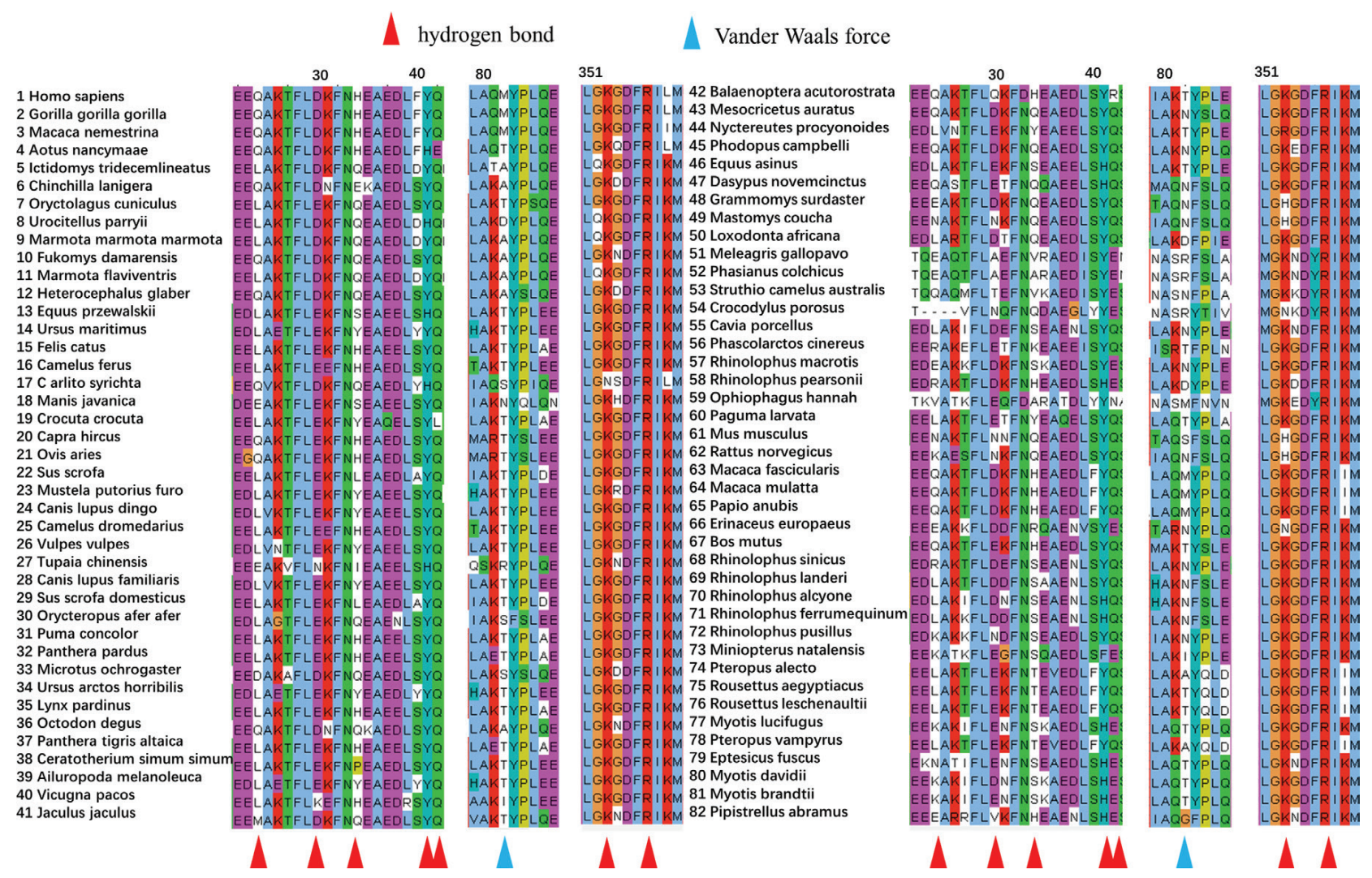

FIGURE 3 | Sequence alignment of ACE2 from 82 species.

ACE2 amino acids binding the SARS-CoV-2 spike-RBD through hydrogen bonds are marked with red triangles below, and those binding the spike-RBD through hydrophobic interactions are marked with blue triangles below.

Lys417, Tyr453, Asn501 and Gln498 in the SARS-CoV-2 spike-RBD. Among them, two hydrogen bonds are formed between $G \ln 42$ in hACE2 and Gln498 in the spike-RBD. Moreover, Lys353 and Arg357 in hACE2 interact with Asn501 and Thr500 in the spike protein, respectively, through hydrogen bonds (Fig 2A and B). In addition, Met82 in ACE2 interacts with Phe486 in the spike-RBD through hydrophobic interactions. We also analyzed the binding pattern of ACE2 from Rhinolophus sinicus and Mesocricetus auratus with the spike-RBD from SARS-CoV-2 through a docking model. Both also form eight hydrogen bonds. According to the sequence comparison results, two key amino acids in the Rhinolophus sinicus ACE2 sequence differ from the human sequence (Fig 2G). In Rhinolophus sinicus, the ACE2 sequence has Arg24 instead of Gln24, and Ser34 instead of His34. Arg24 and Ser34 interact with Ser477 and Gln493 through hydrogen bonds (Fig 2C, D, G and H). Only one key amino acid in the Mesocricetus auratus ACE2 sequence differs from the human sequence (Fig 2E-H). In Mesocricetus auratus, the ACE2 sequence has Gln34 rather than His34, but Gln34 can also form a hydrogen bonding interaction with Tyr453. However, Gln24 in the Mesocricetus auratus ACE2 forms a hydrogen bonding interaction with Asn487 instead of Gln474. The key interactions between amino acids in ACE2 and the spike-RBD are marked in Fig $2 \mathrm{G}$ and $\mathrm{H}$. The detailed comparison of key amino acids for all 82 ACE2s is shown in Fig 3.

\section{Homology modeling and protein-protein docking calculation}

All ACE2 protein structures were homology modeled with ICM modeling software with the hACE2 structure as the template. The binding free energy was calculated by docking the spike protein of SARS-CoV-2 or other coronaviruses with each ACE2 protein. In most cases, the generated conformation resembling the crystal structure of the hACE2 SARS-CoV-2 RBD complex was the conformation with the minimum energy. The results obtained are shown in Tables 1 and 2 .

As shown in Table 1, among all five closely related SARS family coronaviruses, the SARS-CoV-2 spike RBD appeared to have the strongest affinity for hACE2,

TABLE 1 | Binding free energy of hACE2 with the spike RBD from different coronaviruses, calculated by protein-protein docking.

\begin{tabular}{llll}
\hline No. & Virus name & $\begin{array}{l}\text { RBD similarity to } \\
\text { SARS-CoV-2 }\end{array}$ & $\begin{array}{l}\text { Binding free energy } \\
\text { with hACE2 }\left(\mathrm{kJ}^{\mathrm{mol}}{ }^{-1}\right)\end{array}$ \\
\hline 1 & SARS-CoV-2 & $100 \%$ & -50.1326 \\
2 & Pangolin-CoV GD & $97.1 \%$ & -48.0341 \\
3 & Bat RaTG13 & $89.2 \%$ & -44.9803 \\
4 & Pangolin-CoV GX & $87.1 \%$ & -40.1424 \\
5 & SARS-CoV & $74.6 \%$ & -49.2229 \\
\hline
\end{tabular}


TABLE 2 | Binding free energy of the SARS-CoV-2 RBD, RaTG13 RBD and pangolin-CoV GD RBD with ACE2 from different species, calculated by protein-protein docking.

\begin{tabular}{|c|c|c|c|c|c|c|c|}
\hline No. & Species name & Similarity & $\begin{array}{l}\text { GenBank } \\
\text { accession } \\
\text { number }\end{array}$ & $\begin{array}{l}\text { Binding free energy } \\
\text { with SARS-CoV-2 } \\
\text { RBD }\left(\mathrm{kJ} \cdot \mathrm{mol}^{-1}\right)\end{array}$ & $\begin{array}{l}\text { Binding free energy } \\
\text { with RaTG13 RBD } \\
\left(\mathrm{kJ} \cdot \mathrm{mol}^{-1}\right)\end{array}$ & $\begin{array}{l}\text { Binding free energy } \\
\text { with pangolin-CoV GD } \\
\text { RBD }\left(\mathrm{kJ} \cdot \mathrm{mol}^{-1}\right)\end{array}$ & $\begin{array}{l}\text { C- } \alpha \text { RMSD } \\
\text { to human } \\
\text { ACE2 }(\AA)\end{array}$ \\
\hline 1 & Homo sapiens & $100 \%$ & NP_001358344.1 & -50.1326 & -44.9803 & -48.0341 & - \\
\hline 2 & Gorilla gorilla & $99.01 \%$ & XP_018874749.1 & -51.5556 & -42.7332 & -44.7128 & 0.198 \\
\hline 3 & Macaca nemestrina & $95.34 \%$ & XP_011733505.1 & -51.5325 & -42.6326 & -44.0687 & 0.193 \\
\hline 4 & Papio anubis & $95.34 \%$ & XP_021788732.1 & -51.5628 & -42.6162 & -44.0165 & 0.193 \\
\hline 5 & Macaca fascicularis & $95.21 \%$ & XP_005593094.1 & -51.5373 & -42.6172 & -44.021 & 0.193 \\
\hline 6 & Macaca mulatta & $95.21 \%$ & ACI04556.1 & -51.5677 & -42.8581 & -44.0623 & 0.193 \\
\hline 7 & Aotus nancymaae & $92.17 \%$ & XP_012290105.1 & -42.8772 & -42.5036 & -41.4306 & 0.237 \\
\hline 8 & Equus przewalskii & $86.90 \%$ & XP_008542995.1 & -48.8959 & -40.1971 & -35.1463 & 0.258 \\
\hline 9 & Ceratotherium simum & $85.77 \%$ & XP_004435206.1 & -48.3243 & -41.1406 & -42.9484 & 0.228 \\
\hline 10 & Panthera tigris ssp. altaica & $85.70 \%$ & XP_007090142.1 & -50.6125 & -40.7855 & -42.2621 & 0.227 \\
\hline 11 & Puma concolor & $85.59 \%$ & XP_025790417.1 & -50.5544 & -40.7563 & -41.496 & 0.226 \\
\hline 12 & Panthera pardus & $85.47 \%$ & XP_019273508.1 & -50.6849 & -41.7507 & -42.4629 & 0.277 \\
\hline 13 & Ictidomys tridecemlineatus & $85.38 \%$ & XP_005316051.3 & -48.8769 & -42.2544 & -44.4516 & 0.278 \\
\hline 14 & Felis catus & $85.22 \%$ & NP_001034545.1 & -48.8741 & -41.5772 & -42.2018 & 0.266 \\
\hline 15 & Lynx pardinus & $85.22 \%$ & VFV30336.1 & -50.6549 & -39.4012 & -40.0497 & 0.243 \\
\hline 16 & Oryctolagus cuniculus & $85.14 \%$ & XP_002719891.1 & -48.5832 & -42.3481 & -44.3818 & 0.240 \\
\hline 17 & Marmota marmota & $84.88 \%$ & XP_015343540.1 & -48.6519 & -43.0272 & -45.7725 & 0.276 \\
\hline 18 & Urocitellus parryii & $84.76 \%$ & XP_026252505.1 & -47.6377 & -41.1093 & -42.6924 & 0.288 \\
\hline 19 & Marmota flaviventris & $84.76 \%$ & XP_027802308.1 & -48.6645 & -41.4861 & -44.3536 & 0.275 \\
\hline 20 & Manis javanica & $84.76 \%$ & XP_017505746.1 & -46.3551 & -43.2112 & -43.5113 & 0.252 \\
\hline 21 & Chinchilla lanigera & $84.72 \%$ & XP_013362428.1 & -43.1693 & -37.1876 & -40.2995 & 0.233 \\
\hline 22 & Fukomys damarensis & $84.72 \%$ & XP_010643477.1 & -42.1498 & -41.4333 & -42.681 & 0.233 \\
\hline 23 & Jaculus jaculus & $84.63 \%$ & XP_004671523.1 & -46.0314 & -44.3856 & -44.9497 & 0.245 \\
\hline 24 & Heterocephalus glaber & $84.60 \%$ & XP_004866157.1 & -42.0874 & -43.38 & -39.5228 & 0.201 \\
\hline 25 & Octodon degus & $84.47 \%$ & XP_023575315.1 & -35.7756 & -37.3313 & -39.9532 & 0.237 \\
\hline 26 & Mesocricetus auratus & $84.26 \%$ & XP_005074266.1 & -50.4353 & -44.7522 & -47.3596 & 0.253 \\
\hline 27 & Arlito syrichta & $84.10 \%$ & XP_008062810.1 & -37.8413 & -37.389 & -33.1841 & 0.286 \\
\hline 28 & Canis lupus dingo & $84.01 \%$ & XP_025292925.1 & -40.7918 & -35.2498 & -36.5903 & 0.224 \\
\hline 29 & Nyctereutes procyonoides & $84.01 \%$ & ABW16956.1 & -43.609 & -37.77 & -37.9879 & 0.223 \\
\hline 30 & Ursus maritimus & $83.92 \%$ & XP_008694637.1 & -45.0617 & -33.7685 & -35.7023 & 0.271 \\
\hline 31 & Ursus arctos & $83.88 \%$ & XP_026333865.1 & -45.0899 & -35.5917 & -37.2856 & 0.270 \\
\hline 32 & Vulpes vulpes & $83.63 \%$ & XP_025842512.1 & -45.4803 & -34.3498 & -38.4228 & 0.224 \\
\hline 33 & Microtus ochrogaster & $83.63 \%$ & XP_005358818.1 & -44.1707 & -41.3675 & -42.0092 & 0.224 \\
\hline 34 & Canis lupus familiaris & $83.50 \%$ & NP_001158732.1 & -40.7225 & -38.8909 & -37.9272 & 0.297 \\
\hline 35 & Paguma larvata & $83.48 \%$ & Q56NL1.1 & -49.3514 & -37.1641 & -37.1826 & 0.275 \\
\hline 36 & Equus asinus & $83.40 \%$ & XP_014713133.1 & -48.0456 & -39.2759 & -35.7862 & 0.286 \\
\hline 37 & Ailuropoda melanoleuca & $83.38 \%$ & XP_002930657.1 & -45.2657 & -36.5644 & -38.2467 & 0.294 \\
\hline 38 & Crocuta crocuta & $83.35 \%$ & KAF0878287.1 & -50.1934 & -37.8297 & -27.2922 & 0.245 \\
\hline 39 & Vicugna pacos & $83.35 \%$ & XP_006212709.1 & -44.6744 & -35.3267 & -34.9159 & 0.263 \\
\hline
\end{tabular}


TABLE 2 | (continued)

\begin{tabular}{|c|c|c|c|c|c|c|c|}
\hline No. & Species name & Similarity & $\begin{array}{l}\text { GenBank } \\
\text { accession } \\
\text { number }\end{array}$ & $\begin{array}{l}\text { Binding free energy } \\
\text { with SARS-CoV-2 } \\
\text { RBD }\left(\mathrm{kJ} \cdot \mathrm{mol}^{-1}\right)\end{array}$ & $\begin{array}{l}\text { Binding free energy } \\
\text { with RaTG13 RBD } \\
\left(\mathrm{kJ} \cdot \mathrm{mol}^{-1}\right)\end{array}$ & $\begin{array}{l}\text { Binding free energy } \\
\text { with pangolin-CoV GD } \\
\text { RBD }\left(\mathrm{kJ}^{\mathrm{mol}}{ }^{-1}\right)\end{array}$ & $\begin{array}{l}\text { C- } \alpha \text { RMSD } \\
\text { to human } \\
\text { ACE2 }(\AA)\end{array}$ \\
\hline 40 & Camelus ferus & $83.23 \%$ & XP_006194263.1 & -47.3657 & -38.2449 & -38.8581 & 0.233 \\
\hline 41 & Phodopus campbelli & $82.87 \%$ & АСТ66274.1 & -44.875 & -43.316 & -43.7214 & 0.286 \\
\hline 42 & Mustela putorius & $82.74 \%$ & NP_001297119.1 & -45.3724 & -35.8347 & -38.5731 & 0.229 \\
\hline 43 & Balaenoptera acutorostrata & $82.48 \%$ & XP_028020351.1 & -42.7212 & -38.3849 & -38.1349 & 1.312 \\
\hline 44 & Rattus norvegicus & $82.37 \%$ & NP_001012006.1 & -47.2193 & -39.0555 & -42.0742 & 0.273 \\
\hline 45 & Grammomys surdaster & $82.24 \%$ & XP_028617961.1 & -46.6804 & -42.3484 & -45.3621 & 0.250 \\
\hline 46 & Sus scrofa domesticus & $81.94 \%$ & АСТ66265.1 & -48.9879 & -40.7439 & -38.6853 & 0.297 \\
\hline 47 & Mus musculus & $81.86 \%$ & NP_001123985.1 & -44.6578 & -38.9799 & -41.2323 & 0.231 \\
\hline 48 & Capra hircus & $81.74 \%$ & NP_001277036.1 & -49.5148 & -47.6838 & -49.4663 & 1.309 \\
\hline 49 & Ovis aries & $81.74 \%$ & XP_011961657.1 & -49.6762 & -43.7355 & -45.024 & 0.532 \\
\hline 50 & Pteropus alecto & $81.49 \%$ & XP_006911709.1 & -47.2126 & -42.4567 & -44.3341 & 1.31 \\
\hline 51 & Mastomys coucha & $81.38 \%$ & XP_031226742.1 & -46.7412 & -39.664 & -42.4614 & 0.267 \\
\hline 52 & Sus scrofa & $81.37 \%$ & NP_001116542.1 & -49.0061 & -41.5093 & -43.5881 & 0.298 \\
\hline 53 & Rhinolophus pearsonii & $81.37 \%$ & ABU54053.1 & -46.2924 & -34.2089 & -36.0739 & 0.306 \\
\hline 54 & Bos mutus & $81.37 \%$ & XP_005903173.1 & -49.4998 & -41.6701 & -35.4578 & 1.31 \\
\hline 55 & Camelus dromedarius & $80.87 \%$ & KAB1253106.1 & -47.28 & -39.7657 & -40.3033 & 0.287 \\
\hline 56 & Rhinolophus macrotis & $80.87 \%$ & ADN93471.1 & -48.9215 & -43.8471 & -42.8564 & 0.307 \\
\hline 57 & Tupaia chinensis & $80.75 \%$ & XP_006164754.1 & -39.509 & -36.6856 & -37.591 & 0.28 \\
\hline 58 & Miniopterus natalensis & $80.75 \%$ & XP_016058453.1 & -43.4486 & -36.8746 & -37.7009 & 0.394 \\
\hline 59 & Rhinolophus sinicus & $80.62 \%$ & ADN93475.1 & -50.4141 & -39.9513 & -42.6029 & 0.313 \\
\hline 60 & Rhinolophus landeri & $80.62 \%$ & ALJ94034.1 & -46.5592 & -38.814 & -41.538 & 0.324 \\
\hline 61 & Pteropus vampyrus & $80.62 \%$ & XP_011361275.1 & -46.333 & -39.0766 & -42.9668 & 0.471 \\
\hline 62 & Loxodonta africana & $80.50 \%$ & XP_023410960.1 & -45.8706 & -38.0833 & -39.9194 & 0.758 \\
\hline 63 & Rhinolophus alcyone & $80.50 \%$ & ALJ94035.1 & -46.4305 & -39.366 & -30.2506 & 0.324 \\
\hline 64 & Rhinolophus ferrumequinum & $80.50 \%$ & ADN93470.1 & -46.4919 & -39.491 & -40.4483 & 0.313 \\
\hline 65 & Eptesicus fuscus & $80.42 \%$ & XP_008153150.1 & -35.0887 & -36.0798 & -31.4181 & 0.559 \\
\hline 66 & Myotis brandtii & $80.37 \%$ & XP_014399782.1 & -46.1067 & -41.6428 & -43.5682 & 0.614 \\
\hline 67 & Rhinolophus pusillus & $80.35 \%$ & ADN93477.1 & -48.041 & -37.6987 & -38.1246 & 0.312 \\
\hline 68 & Myotis lucifugus & $80.25 \%$ & XP_023609437.1 & -44.8588 & -36.6078 & -39.8423 & 0.359 \\
\hline 69 & Cavia porcellus & $79.54 \%$ & АCT66270.1 & -37.9728 & -33.4454 & -35.6179 & 0.275 \\
\hline 70 & Orycteropus afer & $79.38 \%$ & XP_007951028.1 & -46.2635 & -38.5732 & -41.149 & 0.579 \\
\hline 71 & Myotis davidii & $79.15 \%$ & XP_006775273.1 & -46.8656 & -39.3552 & -43.0102 & 0.473 \\
\hline 72 & Rousettus leschenaultii & $79.13 \%$ & ADJ19219.1 & -44.8589 & -37.6318 & -36.5318 & 0.359 \\
\hline 73 & Dasypus novemcinctus & $79.13 \%$ & XP_004449124.1 & -40.5196 & -42.4187 & -44.3923 & 0.934 \\
\hline 74 & Erinaceus europaeus & $79.01 \%$ & XP_007538670.1 & -49.2088 & -41.1565 & -40.376 & 0.278 \\
\hline 75 & Rousettus aegyptiacus & $78.88 \%$ & XP_015974412.1 & -35.4247 & -38.1481 & -40.8802 & 0.352 \\
\hline 76 & Pipistrellus abramus & $76.45 \%$ & АCT66266.1 & -40.3802 & -36.9656 & -38.7944 & 0.615 \\
\hline 77 & Phascolarctos cinereus & $71.48 \%$ & XP_020863153.1 & -36.0763 & -36.9936 & -35.9102 & 0.323 \\
\hline 78 & Crocodylus porosus & $67.45 \%$ & XP_019384827.1 & -40.4653 & -41.7424 & -32.9734 & 0.688 \\
\hline
\end{tabular}


TABLE 2 | (continued)

\begin{tabular}{|c|c|c|c|c|c|c|c|}
\hline No. & Species name & Similarity & $\begin{array}{l}\text { GenBank } \\
\text { accession } \\
\text { number }\end{array}$ & $\begin{array}{l}\text { Binding free energy } \\
\text { with SARS-CoV-2 } \\
\text { RBD }\left(\mathrm{kJ} \cdot \mathrm{mol}^{-1}\right)\end{array}$ & $\begin{array}{l}\text { Binding free energy } \\
\text { with RaTG13 RBD } \\
\left(\mathrm{kJ} \cdot \mathrm{mol}^{-1}\right)\end{array}$ & $\begin{array}{l}\text { Binding free energy } \\
\text { with pangolin-CoV GD } \\
\text { RBD }\left(\mathrm{kJ} \cdot \mathrm{mol}^{-1}\right)\end{array}$ & $\begin{array}{l}\text { C- } \alpha \text { RMSD } \\
\text { to human } \\
\text { ACE2 }(\AA)\end{array}$ \\
\hline 79 & Phasianus colchicus & $66.09 \%$ & XP_031451919.1 & -36.1372 & -31.6362 & -33.6769 & 1.155 \\
\hline 80 & Struthio camelus & $65.01 \%$ & XP_009667495.1 & -45.8706 & -38.8162 & -35.3141 & 1.142 \\
\hline 81 & Ophiophagus hannah & $56.91 \%$ & ETE61880.1 & -34.6833 & -29.8054 & -31.9762 & 1.112 \\
\hline 82 & Meleagris gallopavo & $55.50 \%$ & XP_019467554.1 & -37.6367 & -38.8765 & -37.4142 & 0.447 \\
\hline
\end{tabular}

in agreement with the observation of the high infectivity of SARS-CoV-2. Although the SARS-CoV RBD has the lowest similarity with that of SARS-CoV-2, its calculated binding affinity was closest to that of SARS-CoV-2. Moreover, slightly different binding modes in the interface were observed, as shown in the complex structures $[14,17]$. For the other three coronaviruses closely related to SARSCoV-2, the greater the similarity of the RBD, the lower the free binding energy. We further chose SARS-CoV-2 and the two most similar viruses, bat RaTG13 and pangolin-CoV GD, for protein-protein docking studies.

According to the results in Table 2 and Fig 4, the binding energy of ACE2 receptors and SARS-CoV-2 RBD in various animals essentially followed a trend in which lower homology with the human sequence was associated with weaker binding energy, with some exceptions.

In primates, because ACE2s are highly homologous to hACE2, they had strong binding energy to RBD, potentially even stronger than that of hACE2. As shown in Table 2, ACE2 from primates (Macaca mulatta, Papio anubis, Gorilla gorilla, Macaca fascicularis and Macaca nemestrina) showed stronger binding to the RBD of SARS-CoV-2 than that hACE2 $\left(-50.1326 \mathrm{~kJ} \cdot \mathrm{mol}^{-1}\right)$, with a lower free binding energy than $-51 \mathrm{~kJ} \cdot \mathrm{mol}^{-1}$. An exception was Aotus nancymaae ACE2, which has $92.17 \%$ sequence similarity to hACE2, but its binding toward the SARS-CoV-2 RBD was significantly lower than that of hACE2 and was also lower than that of some bird ACE2 isoforms.

The ACE2 of most of the Felidae selected in this study, such as Panthera pardus, Lynx pardinus, Panthera tigris and Puma concolor, had stronger binding to the RBD of SARSCoV-2 than that of hACE2, and showed a free binding energy lower than $-50.1326 \mathrm{~kJ} \cdot \mathrm{mol}^{-1}$. However, domestic cat ACE2 had a slightly higher free binding energy than that of hACE2, with a value of $-48.8741 \mathrm{~kJ} \cdot \mathrm{mol}^{-1}$. Notably, the ACE2 of Canidae, including domestic dogs, and also Mustela putorius, had much higher free binding energy than that of hACE2, thus indicating much weaker binding.

However, as shown in Table 2, in species more distantly related to humans, including Mesocricetus auratus and Crocuta crocuta, the ACE2 receptors showed stronger binding toward the RBD of SARS-CoV-2 than did hACE2. The sequence

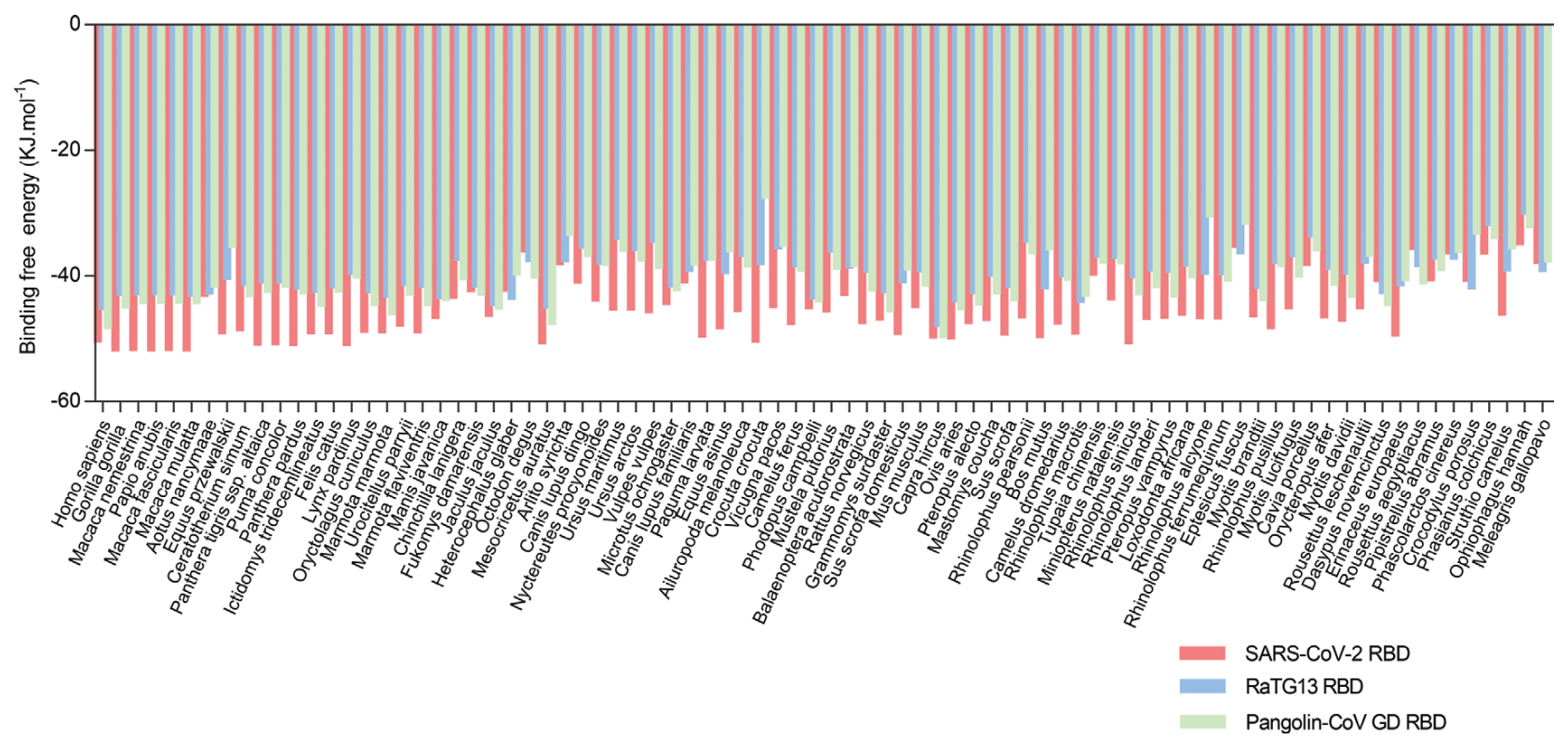

FIGURE 4 | Binding free energy of the SARS-CoV-2 RBD, RaTG13 RBD and pangolin-CoV GD RBD with ACE2 from different species, calculated by protein-protein docking.

Red columns represent SARS-CoV-2 RBD, blue columns represent RaTG13 RBD, and green columns represent pangolin-CoV GD. The closer to the right of the $\mathrm{X}$ axis, the lower the homology with hACE2. 
similarity of Rodentia ACE2s and hACE2 is essentially $81-86 \%$, and in rats and mice, the ability of ACE2 to bind the RBD was significantly weaker than that in humans; however golden hamster ACE2 had greater binding ability than hACE2.

Paguma larvata was confirmed to be the main intermediate host of SARS-CoV [1]. Our prediction results showed that Paguma larvata and Erinaceus europaeus ACE2 had similar binding ability to that of hACE2, thus indicating that these two species are susceptible to SARS-CoV-2. Erinaceus europaeus ACE2 has only $79.01 \%$ sequence similarity with hACE2, but its binding ability to the RBD was very close to that of hACE2.

Rhinolophus pearsonii and Rhinolophus macrotis, belonging to Rhinolophidae, have $81.37 \%$ and $80.87 \%$ sequence similarity with hACE2, but had a binding ability toward RBD similar to that of hACE2. Rhinolophus sinicus ACE2 shares $80.62 \%$ sequence similarity with hACE2, but its binding ability to RBD was stronger than that of hACE2.

These findings suggest that SARS-CoV-2 is similar to bat coronavirus and has the most similar codon usage bias to that of snake coronavirus [14]. However, much controversy exists regarding this conclusion. Therefore, we focused on the possibility of non-mammals as intermediate hosts. As shown in Table 2, the sequence similarity of non-mammalian (Phasianidae, Struthionidae, Elapidae, Phasianidae) ACE2 and hACE2 was only 55-66\%, and all had weak binding ability toward the SARS-CoV-2 RBD.These results indicated that non-mammals (reptiles and birds) cannot be the intermediate hosts of SARS-CoV-2.

To better compare the natural and intermediate hosts of the bat coronavirus RaTG13 and the human SARS-CoV-2 virus, we docked the spike RBD of the bat coronavirus RaTG13 with ACE2 protein from different species and calculated the binding free energy (Table 2). The binding energy of RaTG13 RBD to ACE2 from various animals was similar to that of human SARS-CoV-2 virus (Table 2). Moreover, the binding ability of ACE2 toward the RaTG13 $\mathrm{RBD}$ in almost all species was weaker than that toward SARS-CoV-2, in agreement with previously reported research [18]. The results showed that the sequence similarity of Capra hircus ACE2 to hACE2 is $81.74 \%$, and its binding ability toward RaTG13 RBD was even stronger than that of hACE2. In addition, ACE2 of Rhinolophus macrotis has $80.87 \%$ sequence similarity with hACE2, but its binding ability to RaTG13 RBD was comparable to that of hACE2. Therefore, Rhinolophus macrotis may be the intermediate host of RaTG13. In addition, Mesocricetus auratus, Jaculus jaculus, Ovis aries, Heterocephalus glaber and Phodopus campbelli had strong binding energy toward the RBD of RaTG13.

Further analysis of the binding ability of ACE2 from various animals toward the RBD of pangolin-CoV GD was performed (Table 2), and we found that Capra hircus, Mesocricetus auratus, Homo sapiens and most primates, Marmota marmot, Ictidomys tridecemlineatus, Oryctolagus cuniculus, Marmota flaviventris, Jaculus jaculus, Phodopus campbelli, Ovis aries, Grammomys surdaster, Pteropus alecto, Sus scrofa, Dasypus novemcinctus and Myotis brandtii ACE2 had stronger binding ability toward the RBD of pangolin-CoV GD than that of Manis javanica, the putative intermediate host of SARSCoV-2, particularly Capra hircus. These species may also be susceptible to pangolin-CoV GD.

\section{DISCUSSION}

The COVID-19 pandemic caused by the novel coronavirus SARS-CoV-2 has spread worldwide. Viruses cannot grow and replicate independently, and can replicate themselves only in a host's living cells. Previously, researchers have suggested that bats might be natural hosts of SARS-CoV-2, and snakes, pangolins, turtles and minks might be potential intermediate hosts $[7-9,12-13]$. It is important to find the intermediate host of SARS-CoV-2 to enable the source to be cut off and prevent the virus from being transmitted to humans. However, to confirm the intermediate host, a rigorous scientific process is necessary, as follows: (1) a virus that can reproduce continuously in the intermediate host must be isolated; (2) the disease and pathological characteristics of the isolated virus must be confirmed in animal models; (3) the position of the intermediate host in the infection transmission chain must be confirmed. At present, the intermediate host of SARS-CoV-2 is uncertain.

The binding affinity of the SARS-CoV-2 spike to hACE2 and the ability to escape host immune attack are prerequisites for cross-species transmission to humans. The interaction between the spike protein and ACE2, the first step in viral invasion of the host, directly determines the host range and tissue specificity. To explore possible susceptible animals and intermediate hosts of SARS-CoV-2, we selected 82 representative ACE2 sequences from the 1000 sequences with the closest homology to the hACE2 protein. Most of these species were mammals, and some were birds and reptiles. Through sequence alignment and phylogenetic tree analysis, these species were divided into different families, and the ACE2 proteins of all species were subjected to homology modeling. The spike RBD of SARS-CoV-2 was docked with different ACE2 proteins, and the binding free energy was calculated. The results showed that lower the homology between the ACE2 of the different species and hACE2 was associated with weaker binding ability of the ACE2 receptor to the RBD domain of SARS-CoV-2.

Previous studies have suggested that SARS-CoV-2 might have originated in bats $[6,7]$. We found that Rhinolophus sinicus ACE2 had slightly stronger binding than hACE2 to the SARS-CoV-2 RBD. This result suggests that Rhinolophus sinicus might be susceptible to SARS-CoV-2 and could even be the intermediate host, in agreement with a suggestion by a previous study [6].

Our results showed that the ACE2 of most primates, Crocuta crocuta, Mesocricetus auratus and wild felines had stronger binding to the RBD domain of SARS-CoV-2 than did hACE2, thus implying that these animals might be intermediate hosts of SARS-CoV-2. Most primates, including Gorilla gorilla, Macaca nemestrina, Macaca fascicularis, Macaca 
mulatta (Rhesus macaques) and Papio anubis are suggested to be susceptible to SARS-CoV-2, in agreement with findings from a previous study indicating that conjunctival infection of SARS-CoV-2 can cause mild COVID-19 in rhesus monkeys [19]. We found that the Aotus nancymaae ACE2 has high homology with hACE2, but its binding ability toward the SARS-CoV-2 RBD was much lower than that of hACE2 and even lower than that of ACE2 from some birds. This finding might have been due to the replacement of Tyr41 and Gln 42 of hACE2 by His 41 and Glu42 in Aotus nancymaae (Fig 3). Tyr41 of hACE2 forms hydrogen bonds with Thr500 and Asn501 of the SARS-CoV-2 RBD. Moreover, Glu42 forms hydrogen bonds with Gly446 and Tyr449 of the SARS-CoV-2 RBD. Tyr41 and Gln42 are also highly conserved in other species [20]. The differences in the Aotus nancymaae ACE2 might disrupt the hydrogen-bonding interactions and affect the binding affinity toward the SARS-CoV-2 RBD. In one study, the New World monkey ACE2 with His41 and Glu42 has shown limited ability to mediate SARS-CoV-2 entry, in agreement with our conclusions [21]. Tyr41 and Gln42 of ACE2 are critical to SARS-CoV-2 host range and susceptibility.

On the basis of our findings, most wild felines are likely to be susceptible to SARS-CoV-2. However, given the relatively unlikely contact between wild felines and humans, they are unlikely to be intermediate hosts. Domestic cat (Felis catus) ACE2 showed weaker binding than that of wild felines, but because its free energy remained close to that of hACE2, cats might be susceptible to SARS-CoV-2. In one study, ectopic expression of homologous ACE2 in A549 cells infected with SARS-CoV-2 was used to evaluate the binding affinity to ACE2. Cat ACE2 has shown a strong ability to mediate viral entry [21]. Another study has evaluated the invasion and replication of SARS-CoV-2 in cats and found that SARS-CoV-2 can effectively replicate in cats and spread through the air [22]. These findings indicate that cats are highly sensitive to SARS-CoV-2. Felidae with higher binding affinity ACE2, such as Panthera pardus, Lynx pardinus, Panthera tigris and Puma concolor, might also be susceptible to SARS-CoV-2. In contrast, dogs appear to be much less susceptible, in agreement with findings from previous studies [21-23].

ACE2 in animals such as Paguma larvata, Erinaceus europaeus, Erinaceus europaeus, Bos mutus, Ovis aries, Capra hircus and Sus scrofa had slightly higher binding energy toward SARS-CoV-2 RBD than did hACE2. Because the values were very close, we speculate that these animals might be susceptible to SARS-CoV-2 and could all be potential intermediate hosts.

A recent study has shown that the RBD of pangolin-CoV GD and SARS-CoV-2 is highly conserved, with a difference of only one amino acid, thus suggesting that Manis javanica could be the intermediate host of SARS-CoV-2 $[7,9]$. However, our docking results showed that the binding affinity of SARS-CoV-2 RBD to pangolin ACE2 was not as strong as that of hACE2. Furthermore, analysis of the binding ability of ACE2 from various animals to the RBD of
pangolin-CoV GD revealed that the ACE2 of humans and many animals other than Manis javanica, such as Capra hircus, Mesocricetus auratus, and Marmota marmota, had stronger binding ability to the RBD of pangolin-CoV GD, particularly Capra hircus. This means that pangolin-CoV GD may be able to invade other species besides pangolin. Although pangolins might not be direct intermediate hosts of SARS$\mathrm{CoV}-2$, the high homology of the RBD domain between SARS-CoV-2 and pangolin-CoV GD suggests that pangolin-CoV GD might still be the intermediate virus linking SARS-CoV-2 and its earlier variants. Some studies have also suggested that SARS-CoV-2 might have integrated multiple viruses during its evolution, and pangolins might have been an intermediate host of SARS-CoV-2 [24]. We speculate that pangolin-CoV GD might have evolved with mutations when it spread in its intermediate host, then gained the ability to infect humans and primates.

This present study and previous research [21] together indicate that ferret ACE2 has significantly strong binding energy toward the SARS-CoV-2 RBD. Interestingly, ferrets are susceptible to SARS-CoV-2 without developing severe disease [21,22]. Some previous articles have claimed that snakes might be an intermediate host [25], but this conclusion lacks consideration. Because synonymous codon usage bias analysis is not suitable for studying coronavirus hosts. Our results indicated that ACE2 in reptiles, such as Ophiophagus Hannah and Crocodylus porosus, and birds, such as Phasianus colchicus and Meleagris gallopavo, showed significantly lower binding toward the SARS-CoV-2 spike RBD than mammalian ACE2; therefore, they are unlikely to be the intermediate hosts of SARS-CoV-2.

From the perspective of experimental animals, ferrets, guinea pigs and wild murine species are not good models of SARS-CoV-2, because their ACE2 showed much lower binding to the spike RBD than did hACE2. Primates and golden Syrian hamsters are more suitable experimental animals to serve as SARS-CoV-2 infection models. Existing studies have indicated that golden Syrian hamsters are susceptible to SARS-CoV-2 and exhibit pathological features similar to those of mild human infections. Thus, the golden Syrian hamster might be a potential animal model for studying SARS-CoV-2 spread, pathogenesis, drug and vaccine development $[26,27]$. Our results also indicated that ACE2 in mice, rats, and dogs had weaker affinity for the SARSCoV-2 RBD, and thus these animals are not suitable SARS$\mathrm{CoV}-2$ research models. This conclusion is consistent with the results of a study showing that dogs are not susceptible to SARS-CoV-2 [22]. Because of the low binding efficiency of murine ACE2 to the SARS-CoV-2 spike, the virus cannot easily enter murine cells and cause similar symptoms to those in humans. Attempts have been made to develop transgenic mice expressing hACE2 for SARS-CoV-2 research, but some problems still exist, such as low hACE2 expression, limited tissue distribution and low lethality [28].

Investigations on SARS-CoV-2 susceptible animals that are in close contact with humans are aiming not only to find potential intermediate hosts but more importantly 
to block the cross-species transmission of SARS-CoV-2 and cut off the bidirectional spread and evolution. SARS$\mathrm{CoV}-2$, which broke out among farmed minks last year, first infected humans before spreading and evolving in minks, and it has continued to spread among humans [29]. Recent research has found that wild white-tailed deer in the northeastern United States have been infected with SARS$\mathrm{CoV}-2$, which has spread among deer herds. This is the first report that wild animals have been widely exposed to, and have spread, SARS-CoV-2, but how the virus spread to deer and whether it will continue to spread to other wild species are unknown [30]. Such cross-species transmission and evolution is very dangerous, because humans might have difficulty escaping the coronavirus and could be affected for a long time. Especially, when animals that are in close contact with humans, such as cats and minks, can be infected by SARS-CoV-2.

\section{CONCLUSIONS}

The traceability of SARS-CoV-2 is an extremely important but challenging task. Identifying intermediate hosts and natural hosts is time consuming but necessary to answer a series of questions about how SARS-CoV-2 evolved, how it spread to humans, and how to obtain adaptive mutations, how to increase the affinity with the host receptor, and how to evade the host immune response. Without answers to these questions, discovering SARS-CoV-2 susceptible species and preventing the virus from spreading to humans would be impossible. Our work preliminarily predicts the susceptibility of different species to SARS-CoV-2 by calculating the binding affinity of RBD and ACE2. The susceptible animals predicted herein are consistent with the currently identified SARS-CoV-2 infected animals, such as cats, minks, lions and tigers. In addition, most primates, spotted hyenas, golden Syrian hamsters, hedgehogs and sheep might be susceptible to SARS-CoV-2. These results emphasize the need to continue and expand wildlife surveillance to avoid widespread cross-species viral transmission and evolution. When necessary, rigorous zoonotic disease surveillance plans should be formulated to clarify how the pathogen adapts, evolves and spreads when it invades a new host. From a long-term perspective, we must continue to establish and perfect the strategy of prevention, control and treatment of zoonotic infectious diseases and prepare for the next potential pandemics.

\section{FUNDING}

We are very grateful to the Protein Data Bank, DTU Health Tech, ZINC database and researchers for making data freely available. We acknowledge support from National Mega-project for Innovative Drugs (grant number 2019ZX09721001-004-007), National Natural Science Foundation of China (NSFC) (grant numbers U1803122, 81773637, 81773594), the Fundamental Research Fund for the Central Universities (HUST COVID-19 Rapid Response Call, No. 2020 kfyXGYJ037), fellowship of China Postdoctoral Science Foundation (grant number 2020T130039ZX), Wuhan COVID-19 Emergency Research Fund (grant number EX20C02) and Liaoning Revitalization Talents Program (No. XLYC1807182).

\section{COMPETING INTERESTS}

The authors declare that they have no competing interests.

\section{REFERENCES}

1. Guan $Y$, Zheng BJ, He YQ, Liu XL, Zhuang ZX, Cheung CL, et al. Isolation and characterization of viruses related to the SARS coronavirus from animals in southern China. Science. 2003;302(5643):276-278.

2. Drosten C, Kellam P, Memish ZA. Evidence for camel-tohuman transmission of MERS coronavirus. N Engl J Med. 2014;371(14):1359-1360.

3. Li W, Shi Z, Yu M, Ren W, Smith C, Epstein JH, et al. Bats are natural reservoirs of SARS-like coronaviruses. Science. 2005;310(5748):676-679.

4. Hu B, Zeng LP, Yang XL, Ge XY, Zhang W, Li B, et al. Discovery of a rich gene pool of bat SARS-related coronaviruses provides new insights into the origin of SARS coronavirus. PLoS Pathog. 2017;13(11):e1006698.

5. Yang Y, Du L, Liu C, Wang L, Ma C, Tang J, et al. Receptor usage and cell entry of bat coronavirus HKU4 provide insight into bat-to-human transmission of MERS coronavirus. Proc Natl Acad Sci U S A. 2014;111(34):12516-12521.

6. Zhou P, Yang XL, Wang XG, Hu B, Zhang L, Zhang W, et al. A pneumonia outbreak associated with a new coronavirus of probable bat origin. Nature. 2020;579(7798):270-273.

7. Lam TT, Shum MH, Zhu HC, Tong YG, Ni XB, Liao YS, et al. Identifying SARS-CoV-2 related coronaviruses in Malayan pangolins. Nature. 2020;583(7815):282-285.

8. Zhang T, Wu QF, Zhang ZG. Probable pangolin origin of SARSCoV-2 associated with the COVID-19 outbreak. Curr Biol. 2020;30(7):1346.e2-1351.e2.

9. Xiao KP, Zhai JQ, Feng YY, Zhou N, Zhang X, Zou J-J, et al. Isolation and characterization of 2019-nCoV-like coronavirus from Malayan Pangolins. bioRxiv. 2020. DOI: 10.1101/2020.02.17.951335.

10. Liu P, Jiang JZ, Wan XF, Hua Y, Li L, Zhou J, et al. Are pangolins the intermediate host of the 2019 novel coronavirus (SARS(oV-2)? PLoS Pathog. 2020;16(5):e1008421.

11. Wu C, Zheng M, Yang Y, Gu X, Yang K, Li M, et al. Furin: a potential therapeutic target for COVID-19. iscience. 2020;23(10):101642.

12. Guo Q, Li M, Wang CH, Wang PH, Fang ZC, Tan J, et al. Host and infectivity prediction of Wuhan 2019 novel coronavirus using deep learning algorithm. bioRxiv. 2020. DOI: 10.1101/2020.01.21.914044.

13. Liu Z, Xiao X, Wei X, Li J, Yang J, Tan H, et al. Composition and divergence of coronavirus spike proteins and host ACE2 receptors predict potential intermediate hosts of SARS-CoV-2. J Med Virol. 2020;92(6):595-601.

14. Wang QH, Zhang YF, Wu LL, Niu S, Song CL, Zhang ZY, et al. Structural and functional basis of SARS-CoV-2 entry by using human ACE2. Cell. 2020;181(4):894.e9-904.e9.

15. Abagyan R, Totrov M, Kuznetsov D. ICM-a new method for protein modeling and design applications to docking and structure prediction from the distorted native conformation. J Comput Chem. 1994;15:488-506.

16. Zhou P, Yang XL, Wang XG, Hu B, Zhang L, Zhang W, et al. A pneumonia outbreak associated with a new coronavirus of probable bat. Nature. 2020;579(7798):270-273.

17. Yan RH, Zhang YY, Li YN, Xia L, Guo YY, Zhou Q. Structural basis for the recognition of the SARS-CoV-2 by full-length human ACE2. Science. 2020;367(6485):1444-1448.

18. Liu K, Pan X, Li L, Yu F, Zheng A, Du P, et al. Binding and molecular basis of the bat coronavirus RaTG13 virus to ACE2 in humans and other species. Cell. 2021;184(13):3438 e10-3451.e10. 
19. Deng W, Bao L, Gao H, Xiang Z, Qu Y, Song Z, et al. Ocular conjunctival inoculation of SARS-CoV-2 can cause mild COVID-19 in rhesus macaques. Nat Commun. 2020;11(1):4400.

20. Lan J, Ge J, Yu J, Shan S, Zhou H, Fan S, et al. Structure of the SARS-COV-2 spike receptor-binding domain bound to the ACE2 receptor. Nature. 2020;581(7807):215-220.

21. Liu Y, Hu G, Wang Y, Ren W, Zhao X, Ji F, et al. Functional and genetic analysis of viral receptor ACE2 orthologs reveals a broad potential host range of SARS-CoV-2. Proc Natl Acad Sci U S A. 2021;118(12):e2025373118.

22. Shi J, Wen $Z$, Zhong $G$, Yang $H$, Wang $C$, Huang $B$, et al. Susceptibility of ferrets, cats, dogs, and other domesticated animals to SARS-coronavirus- 2 . Science. 2020;368(6494):1016-1020

23. Zhang Q, Zhang HJ, Huang K, Yang Y, Hui XF, Gao JD, et al. SARS-CoV-2 neutralizing serum antibodies in cats: a serological investigation. Preprint at bioRxiv. 2020. DOI:10.1101/2020.04.01.021196.

24. Xiao K, Zhai J, Feng Y, Zhou N, Zhang X, Zou JJ, et al. Isolation of SARS-CoV-2-related coronavirus from Malayan pangolins. Nature. 2020;583(7815):286-289.
25. Ji W, Wang W, Zhao $X$, Zai J, Li X. Cross-species transmission of the newly identified coronavirus 2019-nCoV. J Med Virol. 2020;92(4):433-440.

26. Sia SF, Yan LM, Chin AWH, Fung K, Choy KT, Wong AYL, et al. Pathogenesis and transmission of SARS-CoV-2 in golden hamsters. Nature. 2020;583(7818):834-838.

27. Chan JF, Zhang AJ, Yuan S, Poon VK, Chan CC, Lee AC, et al. Simulation of the clinical and pathological manifestations of coronavirus disease 2019 (COVID-19) in a golden syrian hamster model: implications for disease pathogenesis and transmissibility. Clin Infect Dis. 2020;71(9):2428-2446.

28. Bao L, Deng W, Huang B, Gao H, Liu J, Ren L, et al. The pathogenicity of SARS-CoV-2 in hACE2 transgenic mice. Nature. 2020;583(7818):830-833.

29. Oude Munnink BB, Sikkema RS, Nieuwenhuijse DF, Molenaar RJ, Munger E, Molenkamp R, et al. Transmission of SARS-CoV-2 on mink farms between humans and mink and back to humans. Science. 2021;371(6525):172-177.

30. Chandler JC, Bevins SN, Ellis JW, Linder TJ, Tell RM, Jenkins-Moore $\mathrm{M}$, et al. SARS-CoV-2 exposure in wild white-tailed deer (Odocoileus virginianus). BioRxiv. DOI: $10.1101 / 2021.07 .29 .454326$. 